\title{
Copy number variation regions in Nellore cattle: Evidences of environment adaptation
}

\author{
Marcos Vinicius Antunes de Lemos ${ }^{\mathrm{a}, *, 1}$, Mariana Piatto Berton ${ }^{\mathrm{a}, 1}$, \\ Gregório Miguel Ferreira de Camargo ${ }^{\mathrm{b}, 1}$, Elisa Peripolli ${ }^{\mathrm{a}, 1}$, Rafael Medeiros de Oliveira Silva ${ }^{\mathrm{a}, 1}$, \\ Bianca Ferreira Olivieri $^{\mathrm{a}, 1}$, Aline S.M. Cesar ${ }^{\mathrm{c}, 1}$, Angélica Simone Cravo Pereira ${ }^{\mathrm{d}, 1}$, \\ Lucia Galvão de Albuquerque ${ }^{\mathrm{a}, 1}$, Henrique Nunes de Oliveira ${ }^{\mathrm{a}, 1}$, Humberto Tonhati ${ }^{\mathrm{a}, 1}$, \\ Fernando Baldi ${ }^{\text {a, } 1}$
}

${ }^{a}$ Department of Animal Science, State University of São Paulo, FCAV - UNESP, Via de acesso Prof. Paulo Donato Castellane, Jaboticabal, São Paulo, Brazil

${ }^{\mathrm{b}}$ Universidade Federal da Bahia (UFBA), Departamento de Zootecnia, Salvador, Bahia, Brazil

${ }^{\mathrm{c}}$ Department of Animal Science, University of São Paulo, Piracicaba, São Paulo, Brazil

${ }^{\mathrm{d}}$ University of São Paulo, Faculdade de Medicina Veterinária e Zootecnia, Department of Nutrition and Animal Production, Pirassununga, SP, Brazil

\section{A R T I C L E I N F O}

\section{Keywords:}

CNVR

Natural selection

Structural variation

Nellore

Subspeciation

\begin{abstract}
A B S T R A C T
The aim of the present study was to analyze the distribution of CNV regions (CNVRs) as well as to address hypothesis about the natural/artificial selection process in Nellore cattle. A total of 399,361 CNVs were identified, using the PennCNV algorithm, in 3794 Nellore cattle (Bos taurus indicus) genotyped with the Bovine HD BeadChip array. The default quality control was applied and 2902 samples and 195,873 CNVs remained. The medium CNV length size was 54,744 bp with a maximum of $870,000 \mathrm{bp}$ and a minimum of $3000 \mathrm{bp}$. The CNVRs were generated by overlapping the 195,873 identified CNVs using the CNVRuler program. There was a higher incidence of CNVRs on BTA19 (24.26\%), BTA23 (18.68\%), and BTA25 (18.05\%). The chromosomes that showed a lower incidence of CNVR were BTA29 (1.63\%), BTA13 (9.72\%), and BTA8 (9.72\%). According to the type, $38.5 \%, 28.5 \%$ and $33.0 \%$ of the CNVRs were characterized as insertion, deletion and mixed (insertion and deletion in the same region), respectively. The 9805 CNVR estimated in the present study covered approximately $13.05 \%$ of the cattle genome (UMD_3.1, 2,649,685,063 bp) and overlapped with 5495 genes. These genes have functions described as involved in biological processes that might be related to the environmental adaptation of the subspecies to tropical areas, such as regulation of vasodilatation, immune system response, hair follicle morphogenesis, among others. This study confirms the existence of large structural variations in the Nellore cattle genome and contributes to understanding the differences between cattle subspecies. Besides, it can also work as a guideline for future studies in which structural variations are present.
\end{abstract}

\section{Introduction}

The identification of new DNA variations affecting quantitative traits is important for beef cattle genetic improvement trough genomic selection. The sequencing of the bovine genome identified more than 27 millions single nucleotide variants and 3,828,041 insertions and deletions (Stafuzza et al., 2017). In the last years, the focus of the scientific community was to identify SNP markers related to phenotypic variation so as to assess genetic variation for genetic improvement. Several forms of DNA variations, i.e. structural genomic variations, may contribute to phenotypic variation (Redon et al., 2006) since they encompass deletions, inversions, mobile-element transpositions, translocations, and copy number variations (CNVs) (Tattini et al., 2015). The CNVs are defined as a DNA segment with $1 \mathrm{~kb}$ or more in length and it presents a variable number of copies, insertions, deletions, translocations or inversions, when compared to a reference genome (Feuk et al., 2006; Scherer et al., 2007).

Several studies have shown the importance of the CNVs for many traits in domestic animals, like cattle (Liu et al., 2008; Seroussi et al., 2010; Seroussi et al., 2012a, 2012b; Cicconardi et al., 2013;), horses (Rosengren-Pielberg et al., 2008), pigs (Paudel et al., 2013; Paudel et al., 2015), sheep (Fontanesi et al., 2011a; Liu et al., 2013;), chickens

\footnotetext{
* Corresponding author.

E-mail address: marcoslemoszootec@gmail.com (M.V. Antunes de Lemos).

${ }^{1}$ All the authors equally contributed to this work.
} 
(Crooijmans et al., 2013; Wang et al., 2010), and dogs (Alvarez and Akey, 2012; Salmon Hillbertz et al., 2007). A considerable number of $\mathrm{CNV}$ maps in cattle were developed using different methods, such as comparative genomic hybridization arrays (aCGH) (Lai et al., 2005; LaFramboise et al., 2009; Pinto et al., 2011), SNP genotyping arrays (Oliveira Junior et al., 2014; da Silva et al., 2016a; da Silva et al., 2016b) and high-throughput next-generation sequencing (Liu et al., 2010; Hou et al., 2011b; Bickhart et al., 2012;) showing that the same genetic variation may be studied from different source of information.

Despite of having a common ancestor, Bos taurus indicus and Bos taurus taurus cattle have undergone separate evolution for numerous hundred thousand years (Machugh et al., 1997a, 1997b). As a result of their domestication process, Bos taurus indicus or zebu animals can be categorized into tropical cattle, having its own phenotypic and genetic differentiation based on the adaptation and evolutionary traits that they possess (Chan et al., 2010). Thus, the zebu cattle have a better adaptation upon high temperatures and a better ability to carry lower burdens of cattle tick. Brazil is one of the largest Nellore's breeder and also a noticeable genetic exporter to others tropical and subtropical regions, contributing whether through purebred selection within the breed or through crosses with local breeds, mainly with European origin. Due to the importance of the Nellore breed and considering the scarce of genomic characterization studies, the aim of the present study was to analyze the distribution of CNV regions (CNVRs) as well as to address hypothesis about the natural/artificial selection process.

\section{Material and methods}

\subsection{Samples}

A total of 3794 Nellore animals (1759 sires and 2035 dams) were used in the present study. The animals were raised in 10 farms located in the Southeast, Northeast, and Midwest regions of Brazil. The farms belonged to three beef cattle breeding programs (CRV-Paint, DeltaG, and Nellore Qualitas). These breeding programs used as selection criteria growth, finishing, and sexual precocity traits. This study was approved by the ethic committee of the Faculty of Agrarian Sciences and Veterinary, São Paulo State University (UNESP).

\section{2. $C N V$ and $C N V R$ detection}

The animals were genotyped with the Bovine HD BeadChip array (High-Density Bovine Bead Chip - Illumina), that contains 777,962 SNP markers. SNP markers with unknown genomic position, located on sex chromosomes, monomorphic and with excess of heterozygosity were removed, as were those with minor allele frequency (MAF) lower than 0.05 and call rate lower than $90 \%$. Samples were also edited for call rate lower than $90 \%$. The CNVs identification was carried out with the SNP data file from the GenomeStudio 1.0 software. For CNVs detection, the PennCNV algorithm was used (Wang et al., 2007). This software incorporates multiple sources of information based on a hidden Markov model for CNV detection from the high-density genotyping data. The PennCNV is the most commonly used algorithm for CNV studies, since it presents a low rate of false positives (Winchester et al., 2009; Tsuang et al., 2010; Eckel-Passow et al., 2011).

Initially, the PennCNV algorithm was used without any quality control parameter so as to obtain the number of CNVs in overall samples (3974 animals). To maintain the quality of the samples, the default PennCNV's quality control was applied, eliminating samples with standard deviation for LRR (log R ratio) higher than 0.30; BAF (frequency of allele B) higher than 0.05; and a value of the waves factor higher than 0.01 (Liu et al., 2013).

After the CNVs identification, the CNVRs were generated by overlapping the CNV samples using the CNVRuler program (Kim et al., 2012). First, individual CNVs are merged into CNVRs, which are genomic regions covering CNVs overlapping by at least 1 bp (Redon et al., 2006). This process is simple and straightforward, but it may overestimate the size of CNVRs when any of the overlapping CNVs are extremely long. In order to minimize this possibility, the CNVRuler provides the option to assess base-by-base the regional density of the participating CNVs and trim the low-density areas. Genomic areas with density lower than $10 \%$ were excluded ("recurrence 0.1 "). The recurrence trims a CNVR based on its frequency to avoid false positive predictions, and it defines more robust limits of the start and end regions. The option "Gain/Loss separated regions" was applied to evaluate the type of the event (gain, loss) in each region. Overlapping "gain" and "loss" CNVRs were merged into single regions to account for genomic regions in which both events can occur ("mixed" CNVRs).

\subsection{Annotation (genes and enrichment)}

Gene content in CNVRs was assessed thought the Ensembl tool VEP database (Bos taurus genes UMD3.1). The genes overlapping CNVRs were subjected to an enrichment gene ontology (GO) analysis with PANTHER and Database for Annotation, Visualization, and Integrated Discovery (DAVID) v6.8 tool (Huang et al., 2009). The enrichment GO analysis determined whether a particular GO was statistically over-represented by the fold enrichment value $(\geq 2.0)$. The enrichment analysis was carried out for the subset of CNVs whose frequency was higher than $1 \%$.

\subsection{Overlap statistics}

Overlap analysis was carried out using the Bioconductor package regioneR (Gel et al., 2016). This package implements a general framework for testing overlaps of genomic regions based on permutation sampling.

\section{Results}

\subsection{CNV and CNVR discovery}

The CNV calling on the UMD3.1 assembly identified a total of 399,361 CNVs for the 3794 samples. After the PennCNV default quality control filtering, a total of 2902 animals were kept for subsequent analyses. A total of $195,873 \mathrm{CNVs}$ were identified with an average, maximum and minimum length size of 54,744, 870,000, and $3000 \mathrm{bp}$, respectively. The CNV length class size with the highest frequency was between 10 and $30 \mathrm{~kb}$ (Fig. 1).

The CNVRs mean length size was 3,528,473 bp, ranging from 520 to $1,476,546 \mathrm{bp}$. The highest rates of CNVRs coverage were found on BTA19 (24.26\%), BTA23 (18.68\%) and BTA25 (18.05\%), and the lowest on BTA29 (1.63\%), BTA13 (9.72\%), and BTA8 (9.72\%) (Fig. 2).

The CNVRs obtained in this study were compared to previous results also reported in Nellore cattle, and a total of 5155 (52.6\%) CNVRs were identified as overlapping with those reported by Da Silva et al. (2016a, 2016b) (Fig. 3).

A total of 9805 CNVRs scattered in all 29 chromosomes were obtained from the CNVs calls. These CNVRs covered approximately $13.05 \%$ of the total length of the bovine genome (UMD_3.1,

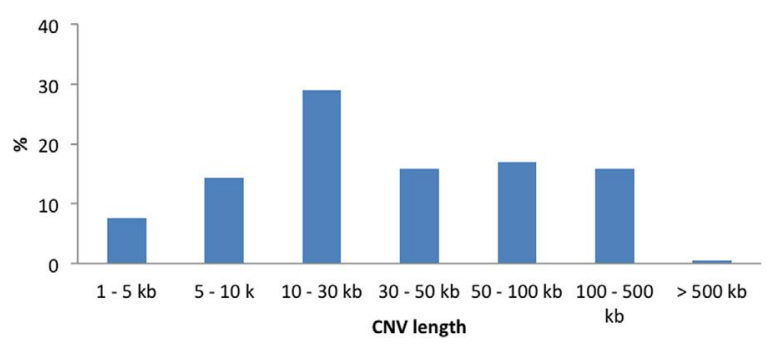

Fig. 1. Range of distribution of CNVs, expressed in kb. 


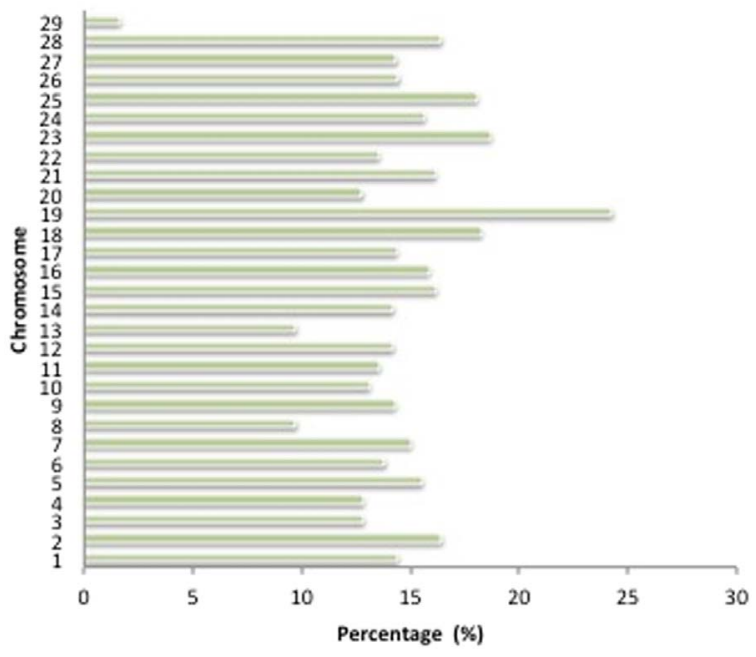

Fig. 2. Percentage of chromosome coverage by CNVRs.

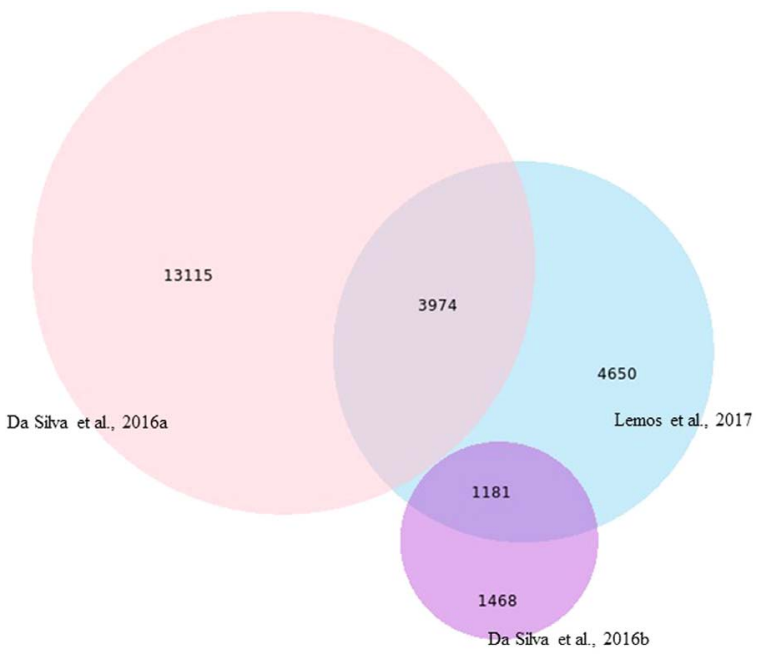

Fig. 3. Venn diagram representing the overlapping CNVRs in Nellore cattle.

2.649.685.063 bp) (Table 1). From the 9805 CNVRs obtained, 38.5\% of them were characterized as insertion regions of copy number, $28.5 \%$ as deletion regions, and $33.0 \%$ showed both events in the same region (insertion and deletion). The BTA28 showed the highest percentage of insertion (47.87\%); meanwhile, the highest percentages of deletion and both events were identified on BTA12 and BTA19, respectively (Fig. 4).

\subsection{Gene content and gene ontology}

The gene content of CNVRs derived from the UMD3.1 autosomes was investigated. The 9805 non-redundant CNVRs identified in the autosomes overlapped with 5495 known genes. Among them, 15\% of the CNVRs were within coding regions, suggesting that exon regions were duplicated or deleted. In addition, $2 \%$ of the CNVRs were in upstream $(1 \%)$ or downstream $(1 \%)$ regions, which were very close to genes regions. Besides, $17 \%$ were in intragenic regions, $19 \%$ of the regions were found in introns and $18 \%$ in non-coding exon regions (5 'and 3' UTR), evidencing the importance the CNVRs (Fig. 5).

The PANTHER accessions were assigned to the overlapped genes for CNVRs identified on UMD3.1. Multiple categories were statistically significant. This set of copy number variable genes encompasses a wide spectrum of molecular functions, biological processes, cellular components, panther protein classes, and pathways. Several GO terms were found to be significantly over-represented, which we highlight
Table 1

CNVR distribution, coverage size and coverage percentage of chromosomes by CNVR detected across Nellore genome.

\begin{tabular}{|c|c|c|c|c|c|}
\hline Chromosome & $\begin{array}{l}\text { Chromosome } \\
\text { size (bp) }\end{array}$ & $\begin{array}{l}\text { Number } \\
\text { of CNVR }\end{array}$ & $\begin{array}{l}\text { CNVR } \\
\text { mean size } \\
\text { (bp) }\end{array}$ & $\begin{array}{l}\text { CNVR } \\
\text { Coverage } \\
\text { (bp) }\end{array}$ & $\%$ Coverage \\
\hline 1 & $158,337,067$ & 732 & $31,496.55$ & $23,055,476$ & 14.56 \\
\hline 2 & $137,060,424$ & 584 & $33,764.03$ & $19,718,197$ & 14.39 \\
\hline 3 & $121,430,405$ & 559 & $29,669.44$ & $15,395,899$ & 12.68 \\
\hline 4 & $120,829,699$ & 515 & $29,891.89$ & $15,394,327$ & 12.74 \\
\hline 5 & $121,191,424$ & 477 & $39,081.58$ & $18,641,916$ & 15.38 \\
\hline 6 & $119,458,736$ & 475 & $34,775.71$ & $16,585,218$ & 13.88 \\
\hline 7 & $112,638,659$ & 469 & $32,827.07$ & $16,518,465$ & 14.67 \\
\hline 8 & $113,384,836$ & 435 & $32,851.60$ & $10,701,231$ & 9.4 \\
\hline 9 & $105,708,250$ & 387 & $33,408.40$ & $14,290,463$ & 13.52 \\
\hline 10 & $104,305,016$ & 385 & $38,737.20$ & $13,107,356$ & 12.57 \\
\hline 11 & $107,310,763$ & 371 & $28,844.28$ & $14,913,824$ & 13.90 \\
\hline 12 & $91,163,125$ & 355 & $31,988.35$ & $11,355,867$ & 12.46 \\
\hline 13 & $84,240,350$ & 352 & $37,525.45$ & 7782,113 & 9.24 \\
\hline 14 & $84,648,390$ & 335 & $33,898.29$ & $11,355,929$ & 13.42 \\
\hline 15 & $85,296,676$ & 316 & $35,129.38$ & $12,929,053$ & 15.16 \\
\hline 16 & $81,724,687$ & 278 & $36,158.40$ & $11,100,887$ & 13.58 \\
\hline 17 & $75,158,596$ & 278 & $32,282.02$ & $10,052,037$ & 13.37 \\
\hline 18 & $66,004,023$ & 272 & $53,521.74$ & $10,949,639$ & 16.59 \\
\hline 19 & $64,057,457$ & 268 & $36,134.05$ & $14,557,915$ & 22.73 \\
\hline 20 & $72,042,655$ & 267 & $41,009.88$ & $8,947,404$ & 12.42 \\
\hline 21 & $71,599,096$ & 226 & $34,434.12$ & $9,683,926$ & 13.53 \\
\hline 22 & $61,435,874$ & 226 & $41,343.97$ & $8,128,130$ & 13.23 \\
\hline 23 & $52,530,062$ & 194 & $32,929.14$ & $9,343,739$ & 17.79 \\
\hline 24 & $62,714,930$ & 194 & $37,253.85$ & $9,388,255$ & 14.97 \\
\hline 25 & $42,904,170$ & 193 & $42,114.66$ & $7,222,553$ & 16.83 \\
\hline 26 & $51,681,464$ & 188 & $34,903.89$ & $7,227,248$ & 13.98 \\
\hline 27 & $45,407,902$ & 177 & $46,287.58$ & $5,736,358$ & 12.63 \\
\hline 28 & $46,312,546$ & 171 & $33,545.95$ & $6,561,933$ & 14.17 \\
\hline 29 & $51,505,224$ & 126 & $57,321.84$ & 819,203 & 1.59 \\
\hline
\end{tabular}

apoptotic process (GO:0006915), biological regulation (GO:0065007), development process (GO:0032502), growth (GO:0040007), immune system process (GO:0002376), metabolic process (GO:0008152), reproduction (GO:0000003), as well as several enriched biological processes.

The same gene dataset was used in the DAVID tool (Huang et al., 2009) so as to analyze the GO functional categories of the protein coding genes located in the CNVs (Table 2). The most enriched GO categories are related to biological regulation process and are shown in Table 2.

\section{Discussion}

A total of $195,873 \mathrm{CNVs}$, with an average length size of 54,744 bp, maximum of 870,000 and a minimum of 3000 bp were identified. The high number and distribution of CNVs in the Nellore genome was also evidenced by Da Silva et al. (2016). These authors detected a total of 68,007 CNVs using the high-density SNP array (BovineHD Genotyping BeadChip) from 1717 Nellore animals. Similarly, Zhou et al. (2016), in a sample of 2230 Nellore cattle identified a total of 992,350 CNVs using a multivariate method supplied by Golden Helix SVS 8.3.0 (Golden Helix, Bozeman, MT, USA http://www.goldenhelix.com).

Several studies have highlighted the distribution and characterization of CNVs in the genome of various animals' species (Fadista et al., 2010; Liu et al., 2010; Hou et al., 2011a). Fadista et al. (2010) reported 304 CNVs regions in 20 animals from Holstein, Simmental, Red Danish, and Hereford breeds. From these preliminary results, new evidences of CNVs in cattle were observed with the use of bovine HapMap (over 500 animals of different breeds), which allowed the identification of 79 candidate deletions (Matukumalli et al., 2009). Bickhart et al., (2012) characterized the genome of three taurine breeds (Angus, Holstein, and Hereford) and one indicine breed (Nellore). The authors observed that the number of CNVRs within the taurine breeds was higher than those 


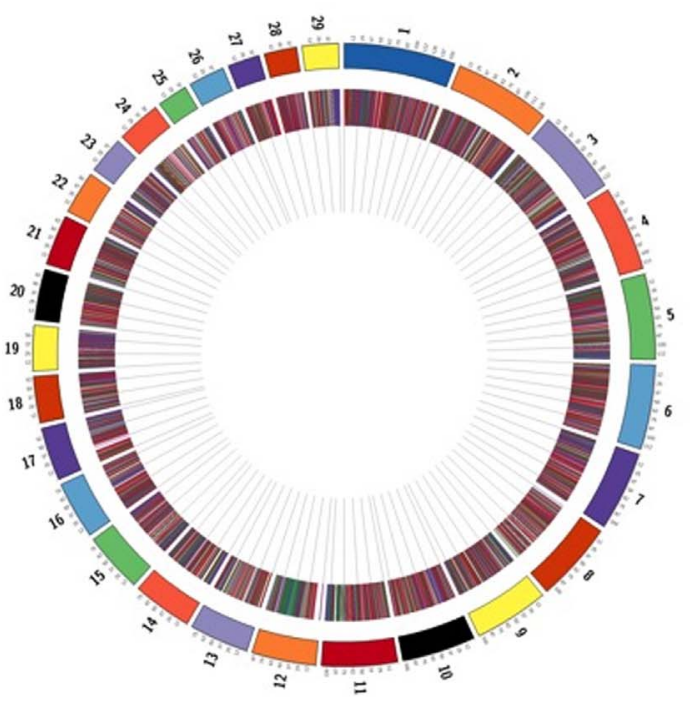

a

28

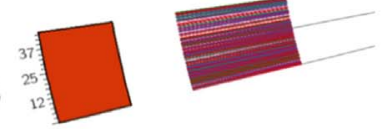

b

12

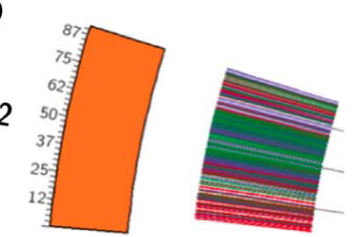

C

19

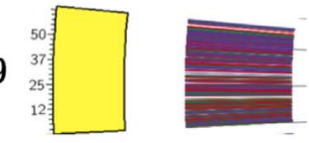

Fig. 4. Comprehensive circular map of autosomal copy-number variants in Bos indicus. From the outside to the inside of the external circle: Chromosome name; genomic location (in Megabases); bars depicting the CNV regions (loss in green, gain in red, and both events in purple). Figs. $4 a, 4 b$ and $4 c$ are a zoom of the chromosome that showed the highest percentage of insertion regions (BTA28, BTA12 and BTA19, respectively). (For interpretation of the references to color in this figure legend, the reader is referred to the web version of this article.)

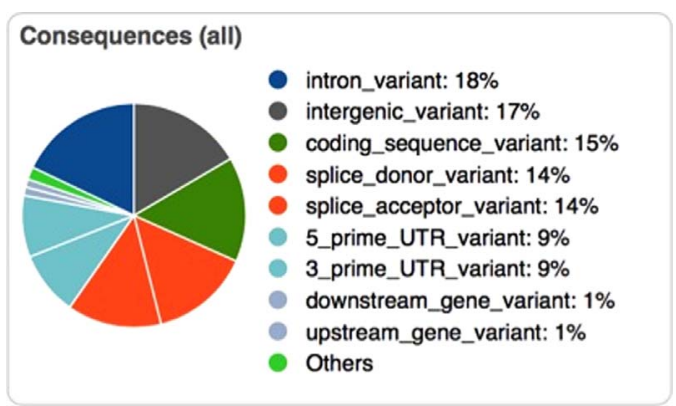

Fig. 5. Graphic representation of CNVRs distribution on the bovine genome.

between taurine and indicine breeds. Moreover, the Nellore breed presented the highest CNVs' diversity among the studied breeds, suggesting that the genetic difference between taurine and indicine breeds might be due to the number of CNVs and its diversity.

By comparing the results discussed above with those of the present study, it possible to note a significant difference in the number of CNVs identified. This divergence may occur due to the use of different methodologies for identifying the CNVs, i.e. different platforms or genotyping algorithms (Table 3). Current methodologies used to infer variations in the number of copies from SNP data tend to lead to some errors at the time of detection (Castellani et al., 2014; Redon et al., 2006). Besides, a number of factors may influence the accuracy of the CNVs detection, such as the breakpoint, the inclusion of batch effects, the stratification of the population, experimental differences, and the robustness of the statistical model (Dellinger et al., 2010). The SNP array used can also interfere with the number of CNVRs discovery, increasing or decreasing the detection sensitivity (Hou et al., 2012).

The proportion of the genome covered by CNVRs was higher than those reported in the literature even for Nellore animals, with values ranging from $0.68 \%$ to $5.85 \%$ (Fadista et al., 2010; Liu et al., 2010; Zhou et al., 2016). Zhang et al. (2014) identified 486 CNVRs covering $2.45 \%$ of the cattle genome in 24 taurine samples, together with 161 CNVRs in two yaks (Bos grunniens) and 163 CNVRs in three buffaloes
(Bubalus bubalis). Hou et al. (2011b) detected 682 CNVRs with $204.4 \mathrm{~kb}$ average in size, covering up to $4.6 \%(139.9 \mathrm{MB})$ of the genome of 21 cattle breeds. The comparison between CNVs and CNVRs is not as easy as it seems since the mapping in each study is based on a different reference genome (Fadista et al., 2010). Notwithstanding the foregoing, a substantial number of CNVRs were overlapped with previous CNVRs identified in Nellore cattle, suggesting that an important portion of the CNVRs were conserved within the Nellore breed over time. Comparing the results obtained in the present study with those presented by Da Silva et al. (2016a, 2016b), 52.6\% of the genomic regions encompassed by the CNVRs (5155 CNVRs) were overlapped in both studies.

The higher proportion of the genome covered by CNVRs in this study may be explained by the large number of samples available for analysis, the methodology applied for identifying the CNV and CNVRs, and also due to the sub-specie (Bos taurus indicus) used in the present study.

Preliminary analysis showed that the number of CNVRs identified increased as the number of samples raised. However, it is important to consider the possibility of a bias due to the reference genome, mainly from Bos taurus taurus cattle. A Bos taurus indicus public reference genome would better guide the indicine genetic studies. Several studies characterizing CNVs in cattle have been performed, and most of them were carried out in taurine (Salomon-Torres et al., 2016; Jiang et al., 2012; Liu et al., 2010) and some in indicine cattle (Zhou et al., 2016; Da Silva et al., 2016a, 2016b). Besides, results have shown that more CNV loci have been reported and predicted in indicine breeds compared to European taurine breeds, which is consistent with the known breed divergence and history (Liu et al., 2010). Recent studies reported that CNVs evolved 2.5 folds faster than SNPs and helped to promote a better adaptation upon different environments (Paudel et al., 2015), being in accordance to the cattle sub-speciation as a result of their domestication process over time.

The relationship between the chromosome size and the number of CNVRs is straightforward (Fig. 6). Although some chromosomes revealed a higher proportion of CNVRs than others (relative to their size), the correlation between the number of detected CNVRs and the chromosome size was 0.96 . This result was in accordance with that reported 
Table 2

Gene ontology enrichment.

\begin{tabular}{|c|c|c|c|c|c|}
\hline Category & Term & Count & P-Value & Fold Enrichment & FDR \\
\hline GOTERM_BP_DIRECT & negative regulation of cAMP-dependent protein kinase activity & 4 & $2,4 \mathrm{E}-2$ & 5,2 & $3,5 \mathrm{E} 1$ \\
\hline GOTERM_BP_DIRECT & histone deubiquitination & 4 & $2,4 \mathrm{E}-2$ & 5,2 & $3,5 \mathrm{E} 1$ \\
\hline GOTERM_BP_DIRECT & regulation of vasodilation & 3 & $9,7 \mathrm{E}-2$ & 5,2 & $8,3 \mathrm{E} 1$ \\
\hline GOTERM_BP_DIRECT & positive regulation of histone methylation & 3 & $9,7 \mathrm{E}-2$ & 5,2 & $8,3 \mathrm{E} 1$ \\
\hline GOTERM_BP_DIRECT & positive regulation of cardiac muscle cell proliferation & 3 & $9,7 \mathrm{E}-2$ & 5,2 & $8,3 \mathrm{E} 1$ \\
\hline GOTERM_BP_DIRECT & protein localization to endosome & 3 & $9,7 \mathrm{E}-2$ & 5,2 & $8,3 \mathrm{E} 1$ \\
\hline GOTERM_BP_DIRECT & regulation of proteasomal ubiquitin-dependent protein catabolic process & 4 & $5,2 \mathrm{E}-2$ & 4,2 & $6,1 \mathrm{E} 1$ \\
\hline GOTERM_BP_DIRECT & cellular response to extracellular stimulus & 4 & $5,2 \mathrm{E}-2$ & 4,2 & $6,1 \mathrm{E} 1$ \\
\hline GOTERM_BP_DIRECT & amino acid transmembrane transport & 4 & $8,9 \mathrm{E}-2$ & 3,5 & $8,1 \mathrm{E} 1$ \\
\hline GOTERM_BP_DIRECT & signal transduction by protein phosphorylation & 8 & $5,5 \mathrm{E}-3$ & 3,2 & $9,3 \mathrm{E} 0$ \\
\hline GOTERM_BP_DIRECT & cellular response to cAMP & 5 & $7,5 \mathrm{E}-2$ & 2,9 & $7,5 \mathrm{E} 1$ \\
\hline GOTERM_BP_DIRECT & somitogenesis & 5 & $7,5 \mathrm{E}-2$ & 2,9 & $7,5 \mathrm{E} 1$ \\
\hline GOTERM_BP_DIRECT & hair follicle morphogenesis & 5 & $7,5 \mathrm{E}-2$ & 2,9 & $7,5 \mathrm{E} 1$ \\
\hline GOTERM_BP_DIRECT & negative regulation of JAK-STAT cascade & 6 & $4,3 \mathrm{E}-2$ & 2,8 & $5,4 \mathrm{E} 1$ \\
\hline GOTERM_BP_DIRECT & retrograde vesicle-mediated transport, Golgi to ER & 7 & $3,6 \mathrm{E}-2$ & 2,6 & $4,8 \mathrm{E} 1$ \\
\hline GOTERM_BP_DIRECT & protein tetramerization & 6 & $8,6 \mathrm{E}-2$ & 2,4 & $7,9 \mathrm{E} 1$ \\
\hline GOTERM_BP_DIRECT & regulation of autophagy & 6 & $8,6 \mathrm{E}-2$ & 2,4 & 7,9E1 \\
\hline GOTERM_BP_DIRECT & negative regulation of cyclin-dependent protein kinase activity & 10 & $1,5 \mathrm{E}-2$ & 2,4 & 2,4E1 \\
\hline GOTERM_BP_DIRECT & protein autophosphorylation & 9 & $3,4 \mathrm{E}-2$ & 2,2 & $4,6 \mathrm{E} 1$ \\
\hline GOTERM_BP_DIRECT & MAPK cascade & 9 & $3,4 \mathrm{E}-2$ & 2,2 & $4,6 \mathrm{E} 1$ \\
\hline GOTERM_BP_DIRECT & fatty acid biosynthetic process & 7 & $9,0 \mathrm{E}-2$ & 2,1 & $8,1 \mathrm{E} 1$ \\
\hline GOTERM_BP_DIRECT & chloride transmembrane transport & 11 & $2,3 \mathrm{E}-2$ & 2,1 & $3,4 \mathrm{E} 1$ \\
\hline GOTERM_BP_DIRECT & cell cycle arrest & 9 & $5,8 \mathrm{E}-2$ & 2,0 & $6,5 \mathrm{E} 1$ \\
\hline GOTERM_BP_DIRECT & heart development & 12 & $2,5 \mathrm{E}-2$ & 2,0 & $3,5 \mathrm{E} 1$ \\
\hline GOTERM_BP_DIRECT & ubiquitin-dependent protein catabolic process & 17 & $1,1 \mathrm{E}-2$ & 1,9 & $1,8 \mathrm{E} 1$ \\
\hline GOTERM_BP_DIRECT & protein stabilization & 12 & $5,8 \mathrm{E}-2$ & 1,8 & $6,5 \mathrm{E} 1$ \\
\hline GOTERM_BP_DIRECT & cell proliferation & 15 & $3,2 \mathrm{E}-2$ & 1,8 & $4,3 \mathrm{E} 1$ \\
\hline GOTERM_BP_DIRECT & transcription from RNA polymerase II promoter & 25 & $7,8 \mathrm{E}-3$ & 1,7 & 1,3E1 \\
\hline GOTERM_BP_DIRECT & negative regulation of apoptotic process & 25 & $2,7 \mathrm{E}-2$ & 1,5 & $3,8 \mathrm{E} 1$ \\
\hline GOTERM_BP_DIRECT & intracellular protein transport & 20 & $6,6 \mathrm{E}-2$ & 1,5 & $7,0 \mathrm{E} 1$ \\
\hline GOTERM_BP_DIRECT & positive regulation of transcription from RNA polymerase II promoter & 50 & $3,0 \mathrm{E}-3$ & 1,5 & $5,1 \mathrm{E} 0$ \\
\hline
\end{tabular}

Gene ontology (GO) categories significantly over represented, with false discovery rate (FDR).

by Da Silva et al. (2016a) for Nellore cattle (0.98). The BTA1 exhibited the highest number of CNVRs (692) and CNVRs mean length size (46.68 kb), while BTA25 the lowest number of CNVRs (131) and CNVRs mean length size $(79.37 \mathrm{~kb})$.

Cicconardi et al. (2013) found 1522 CNVs in Italian Friesian cattle breed, in which $27.52 \%$ of them were insertion and $75.29 \%$ deletion regions. Da Silva et al. (2016a) found 4801 CNVRs of mixed events in the Nellore genome, while the number of deletions and insertions were 212 and 2306, respectively. In the present study, from the total of CNVRs obtained (9805), $38.5 \%$ of them were characterized as insertion regions of copy number, $28.5 \%$ as deletion regions and $33.0 \%$ showed both events in the same region (insertion and deletion). Compared to previous studies (Fadista et al., 2010; Bae et al., 2010; Seroussi et al., 2010; Liu et al., 2010; Hou et al., 2010; Cicconardi et al., 2013; Da Silva et al., 2016b), the results obtained in the present study pointed out the presence of a higher number of CNVRs and insertion regions. The insertion or deletion events of one or more copies of a gene could result in a proportional reduction or absence of the gene product, and it is determined according to the distance that the CNVRs are from the gene (Iafrate et al., 2014). The present study revealed a large proportion of insertion regions, thus, a higher proportion of genes affected by the CNVRs were observed when compared to other studies (Hou et al., 2011a; Bae et al., 2010; Cicconardi et al., 2013).

The GO analyses showed several enriched terms for the CNVRs gene list. One of them was the immune system response (GO:0002376), which is defined as the process involved in the development or functioning of the immune system, i.e. an organismal system for calibrated responses to potential internal or invasive threats. Some authors reported over-represented genes related to the immune system and sensory response in cattle and humans (Nguyen et al., 2006). This fact suggests that the survivability benefit can be achieved with the increased dosage of genes related to the immune and environmental sensory response (Stothard et al., 2011; Liu et al., 2010).

Indicine breeds are favored in tropical regions due to their tolerance to heat and parasites resistance, being the focus of several studies that aims to compare their immunological response upon various environmental factors. In this prerogative, O'Kelly (1980) found higher resistance of indicine crossbreeds raised in tropical conditions, mainly to Haemonchus and Cooperia, when compared to taurine purebred animals. The animal's resistance to internal and external parasites, which stands

Table 3

List of CNVs detection studies in cattle using different approaches.

\begin{tabular}{|c|c|c|c|c|c|}
\hline Study & Method & Algorithm & Samples & Bovine sub-specie & CNV \\
\hline Hou et al. 2012a & SNP chip (777 kb) & PennCNV & 674 & Bos taurus & 443 \\
\hline Coccinard et al., 2013 & SNP chip (648 kb) & PennCNV; QuantiSNP & 2654 & Bos taurus \& Bos indicus & 4839 \\
\hline Salomón-Torres et al. (2015) & SNP chip (648 kb) & PennCNV; QuantiSNP & 12 & Bos taurus & 77 \\
\hline Yang et al., 2015 & SNP chip (770 kb) & PennCNV; CNVPartition & 792 & Bos taurus & 263 \\
\hline Wang et al., 2015 & SNP chip (54 kb) & PennCNV & 231 & Bos taurus $x$ Bos indicus & 433 \\
\hline Zhang et al., 2015 & aCGH & seg-MNT & 24 & Bos taurus \& Bos indicus & 356 \\
\hline Zhou et al. (2016) & 700,000 SNP probes & Golden Helix SVS 8.3.0 & 2230 & Bos indicus & 992,350 \\
\hline Da Silva et al., 2016 & SNP chip (777 kb) & PennCNV; & 1717 & Bos indicus & 68,000 \\
\hline This study & SNP chip (777 kb) & PennCNV; CNVRuler & 3794 & Bos indicus & 195,873 \\
\hline
\end{tabular}




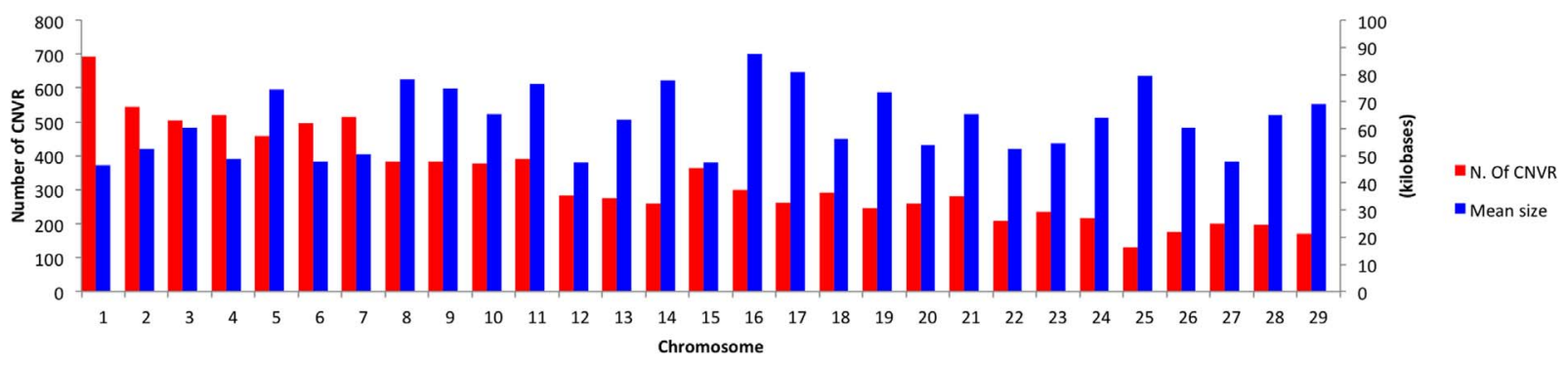

Fig. 6. Number and mean length size of copy number variation regions by chromosome.

for the immune response system efficiency, was "acquired" by natural selection in the Indian subcontinent (O'Kelly, 1980). Up to date, few studies have enlighten the mechanisms underlying the resistance and adaptation traits, thus, the natural selection and domestication process (breed divergence) has been speculated as the start point to explain those acquired traits over time.

The regulation of vasodilatation (GO:0045909) had a 5.2-fold enrichment through DAVID analyzes. This term is defined as any process that modulates the frequency, rate or extent of increases in the diameter of blood vessels. Indicine cattle show a higher thermo tolerance when compared to taurine cattle. Although the physiological basis for this fact has not yet been identified, one possibility is that the density of arteriovenous anastomoses is higher in indicine cattle (Finch, 1985). These structures have lower resistance to flow than vascular passages, it involves capillary networks and facilitates the blood flow to the skin during heat stress (Hales et al., 1978), being a more efficient way to dissipate heat. Another enriched term associated with heat tolerance was hair follicle morphogenesis (GO:0031069) (Finch et al., 1984). The indicine cattle have thin, short, smooth and shiny hair coats. The light color hair reflects a large proportion of solar incident radiation and also acts to reduce heat exchange via radiation (Hutchinson and Brown, 1969; Cardoso et al., 2015). To better dissipate the heat, indicine cattle also have a higher hair angle and a low hair density per skin area (Cruz et al., 2016). All these physiological mechanisms are highly associated with the term pointed out, evidencing its better environmental adaptation.

Terms related to performance were also identified (Panther analyses), such as growth (GO:0040007), defined as the increase in size or mass of an entire organism or a part of an organism. It has been previously reported in a CNV study done by Stothard et al. (2011) with taurine animals. This term could appear due to the selection for weight in Nellore cattle over the last decades; or even due to the lower weight gain that indicine breeds still have when compared to taurine breeds. The term reproduction (GO:0000003) was also significant. It is defined as the production of new individuals that contain some portion of genetic material inherited from one or more parent organisms (Stothard et al., 2011; Ashburner et al., 2000). It is fully described that indicine cattle are outstanding in longevity when compared to taurine breeds, especially regarding their aggregate culling rates for death, unsoundnesses, and injuries. (Rohrer et al., 1988a, 1988b). The CNVRs herein identified may be associated with these phenotypes and differences between subspecies.

Some others relevant biological processes were enriched by the gene list, such as fatty acid biosynthesis process (GO:0006633). For definition, fatty acid biosynthesis are the chemical reactions and pathways resulting in the formation of a fatty acid, any of the aliphatic monocarboxylic acids that can be liberated by hydrolysis from naturally occurring fats and oils (Ashburner et al., 2000). An important group of genes belonging to the ELOVL family are responsible for performing the fatty acids biosynthesis and it has been previously linked to beef fatty acid profile in Nellore cattle (Lemos et al., 2016). The authors, working with the same group of animals of this study, associated the ELVOL5 gene with the arachidonic fatty acid, which is a polyunsaturated fatty acid present in the phospholipids membranes of the body's cell. This fatty acid is abundant in the brain, muscles and liver, and it is the product of their oxygenation and it can mediate or modulate inflammatory reactions (Smith et al., 1979; Samuelson, 1991). The enrichment of these terms could reflect a selection for muscle mass production with improved fatty acid profile, associated with a better resistance of these animals to inflammatory processes.

Biological regulation process (GO:0065007), development process (GO:0032502), apoptotic process (GO:0006915), among others, were enriched in DAVID analyses, further confirming the Panther results. The results of the functional analyses were in agreement with those reported in previous CNV studies in cattle (Fadista et al., 2010; Seroussi et al., 201; Hou et al., 2012; Cicconardi et al., 2013; Da Silva et al., 2016b). Recently, Da Silva et al. (2016a) reported 4097 CNVRs located within genomic regions containing 10,399 annotated genes, revealing important GO categories, i.e. metabolic and cellular processes, biological regulation, immune system process, response to stimulus, cell signaling, reproduction, and growth. Therefore, it is important to emphasize the importance of a deep investigation within these regions, as well as their validation, which can disclose about important mechanisms that underlie and affect economically important traits.

Understanding the genetic basis for productive and functional traits in zebu cattle have a great economic and biological importance. The broad identification of CNVs segregating within the breed provided crucial information to infer that CNVs may be subject to natural and/or artificial selection. The variations in the number of copies of the Nellore cattle genome brings important information to identify genetic variations associated with phenotypes of interest, such as production and meat quality traits, which studies are often restricted to the use of SNPs arrays. The information obtained in this study should support to conduct further association and fine mapping studies that aim to understand the genetic mechanisms and biological pathways underlying the productive and functional traits in Nellore cattle.

\section{Conclusion}

The present study revealed a high variability of CNVs in the Nellore (Bos taurus indicus) cattle genome. The gene enrichment analysis of the CNVRs revealed biological processes that might be involved in the environmental adaptation of the subspecies to tropical areas. In this regard, contributions to understand differences between cattle subspecies, as well as, can be used as s guideline for future studies with structural variations were presented.

\section{Acknowledgments}

MVA Lemos, (FAPESP, Fundação de Amparo à Pesquisa do Estado de São Paulo). F Baldi (FAPESP, Fundação de Amparo à Pesquisa do Estado de São Paulo grant \#2011/21241-0). Lucia G. Albuquerque (FAPESP, Fundação de Amparo à Pesquisa do Estado de São Paulo grant \#2009/16118-5). 


\section{Competing interests}

The authors declare that they have no competing interests.

\section{References}

Alvarez, C.E., Akey, J.M., 2012. Copy number variation in the domestic dog. Mamm. Genome 23 (1-2), 144-163.

Ashburner, M., Ball, C.A., Blake, J.A., Botstein, D., Butler, H., Cherry, J.M., Davis, A.P., Dolinski, K., Dwight, S.S., Eppig, J.T., Harris, M.A., Hill, D.P., Issel-Tarver, L., Kasarskis, A., Lewis, S., Matese, J.C., Richardson, J.E., Ringwald, M., Rubin, G.M., Sherlock, G., 2000. Gene ontology: tool for the unification of biology. Nat. Genet. 25 (1), 25-29.

Bae, J.S., Cheong, H.S., Kim, L.H., Namgung, S., Park, T.J., Chun, J.Y., KIM, J.Y., Charisse, F.A., Lee, J.S., Shin, H.D., 2010. Identification of copy number variations and common deletion polymorphisms in cattle. BMC Genom. 11, 232.

Bickhart, D.M., Hou, Y., Schroeder, S.G., Alkan, C., Cardone, M.F., Matukumalli, L.K., Song, J., Schnabel, R.D., Ventura, M., Taylor, J.F., Garcia, J.F., Van Tassell, C.P., Sonstegard, T.S., Eichler, E.E., Liu, G.E., 2012. Copy number variation of individual cattle genomes using next-generation sequencing. Genome Res. 22, 778-790.

Cardoso, C.C., Peripolli, V., Amador, S.A., Brandão, E.G., Esteves, G.I.F., Sousa, C.M.Z., 2015. França MFMS, Gonçalves FG, Barbosa F.A, Montalvão TC, Martins CF, Fonseca Neto AM, McManus C. Physiological and thermographic response to heat stress in zebu cattle. Livest. Sci. 182, 83-92.

Castellani, C.A., Melka, M.G., Wishart, A.E., Locke, M.E.O., Awamleh, Z., O'Reilly, R.L., 2014. Biological relevance of CNV calling methods using familial relatedness including monozygotic twins. BMC Bioinform. 15, 114.

Chan, E.K.F., Nagaraj, S.H., Reverter, A., 2010. The evolution of tropical adaptation: Comparing taurine and zebu cattle. Anim. Genet. 41 (5), 467-477.

Cicconardi, F., Chillemi, G., Tramontano, A., Marchitelli, C., Valentini, A., AjmoneMarsan, P., 2013. Massive screening of copy number population-scale variation in Bos taurus genome. BMC Genom. 14, 124. http://dx.doi.org/10.1186/1471-2164-14124. (pmid:23442185).

Crooijmans, R.P.M.A., Fife, M.S., Fitzgerald, T.W., Strickland, S., Cheng, H.H., Kaiser, P., Redon, R., Groenen, M.A.M., 2013. Large scale variation in DNA copy number in chicken breeds. BMC Genom. 14, 398.

Cruz, P.F.F., Monteiro, C.P., Guimarães, E.C., Antunes, R.C., MRBM, Nascimento, 2016 Physiological parameters, hair coat morphological characteristics And temperature gradients In Holstein- Gyr crossbred cows. Biosci. J. Uberlândia 32 (2), 471-477.

Da Silva, J.M., Giachetto, P.F., da Silva, L.O., Cintra, L.C., Paiva, S.R., Yamagishi, M.E.B., Caetano, A.R., 2016a. Genome-wide copy number variation (CNV) detection in Nelore cattle reveals highly frequent variants in genome regions harboring QTLs affecting production traits. BMC Genom. 17, 454.

Da Silva, V.H., Regitano, L.C.A., Geistlinger, L., Pértille, F., Giachetto, P.F., Brassaloti, R.A., Morosini, N.S., Zimmer, R., Ll, Coutinho, 2016b. Genome-wide detection of CNVs and their association with meat tenderness in nelore cattl. Plos One 11 (6), e0157711.

Dellinger, A.E., Saw, S.M., Goh, L.K., Seielstad, M., Young, T.L., Li, Y.J., 2010. Comparative analyses of seven algorithms for copy number variant identification from single nucleotide polymorphism arrays. Nucleic Acids Res. 38, e105.

Eckel-Passow, J.E., Atkinson, E.J., Maharjan, S., Kardia, S.L., de Andrade, M., 2011. Software comparison for evaluating genomic copy number variation for Affymetrix 6.0 SNP array platform. BMC Bioinform. 12, 220.

Fadista, J., Thomsen, B., Holm, L.E., Bendixen, C., 2010. Copy number variation in the bovine genome. BMC Genom. 11, 284.

Feuk, L., Carson, A.R., Scherer, S.W., 2006. Structural variation in the human genome. Nat. Rev. Genet. 7, 85-97.

Finch, V.A., Bennett, I.L., Holmes, C.R., 1984. Coat colour in cattle: effect on thermal balance, behaviour and growth, and relationship with coat type. J. Agric. Sci. 1984 (102), 141-147.

Finch, V.A., 1985. Comparison of non-evaporative heat transfer in different cattle breeds. Aust. J. Agric. Res. 36, 497-508.

Fontanesi, L., Beretti, F., Martelli, P.L., Colombo, M., Dall'olio, S., Occidente, M., Portolano, B., Casadio, R., Matassino, D., Russo, V., 2011a. A first comparative map of copy number variations in the sheep genome. Genomics 97, 158-165.

Gel, B., Diez-Villanueva, A., Serra, E., Buschbeck, M., Peinado, M.A., Malinverni, R., 2016. regioneR: an R/Bioconductor package for the association analysis of genomic regions based on permutation tests. Bioinformatics 32 (2), 289-291.

Hales, J.R., Fawcett, A.A., Bennett, J.W., Needham, A.D., 1978. Thermal control of blood flow through capillaries and arteriovenous anastomoses in skin of sheep. Pflug. Arch. 1978 (378), 55-63.

Hou, Y., Bickhart, D.M., Chung, H., Hutchison, J.L., Norman, H.D., Connor, E.E., 2012. Analysis of copy number variations in Holstein cows identify potential mechanisms contributing to differences in residual feed intake. Funct. Integr. Genom. 12, 717-723. http://dx.doi.org/10.1007/s10142-012-0295-y. (pmid:22991089).

Hou, Y., Liu, G.E., Bickhart, D.M., Cardone, M.F., Wang, K., Kim, E., 2011a. Genomic characteristics of cattle copy number variations. BMC Genom. 12, 127. http://dx.doi. org/10.1186/1471-2164-12-127. (pmid:21345189).

Hou, Y., Liu, G.E., Bickhart, D.M., Cardone, M.F., Wang, K., Kim, E.S., Matukumalli, L.K., Ventura, M., Song, J., Vanraden, P.M., 2011b. Genomic characteristics of cattle copy number variations. BMC Genom. 12, 127.

Huang, W., Sherman, B.T., Lempicki, R.A., 2009. Bioinformatics enrichment tools: paths toward the comprehensive functional analysis of large gene lists. Nucleic Acids Res. 37 (1), 1-13.
Jiang, L., Jiang, J., Wang, J., Ding, X., Liu, J., Zhang, Q., 2012. Genome-wide identification of copy number variations in Chinese Holstein. PLoS One 7, e48732. http://dx. doi.org/10.1371/journal.pone.0048732. (pmid:23144949).

Kim, J.H., Hu, H.J., Yim, S.H., Bae, J.S., Kim, S.Y., Chung, Y., 2012. CNVRuler: a copy number variation-based case-control association analysis tool. Bioinformatics 28 (13), 1790-1792.

LaFramboise, T., 2009. Single nucleotide polymorphism arrays: a decade of biological, computational, and technological advances. Nucleic Acids Res 37, 4181-4193.

Lai, W.R., Johnson, M.D., Kucherlapati, R., Park, P.J., 2005. Comparative analysis of algorithms for identifying amplifications and deletions in array CGH data. Bioinformatics 21, 3763-3770.

Lemos, M.V.A., Chiaia, H.L.J., Berton, M.P., Feitosa, F.L.B., Aboujaoud, C., Camargo, G.M.F., Pereira, A.S.C., Albuquerque, L.G., Ferrinho, A.M., Mueller, L.F., Mazalli, M.R., Furlan, J.J.M., Carvalheiro, R., Gordo, D.M., Tonussi, R., Espigolan, R., Silva, R.M.O., De Oliveira, H.N., Duckett, S., Aguilar, I., Baldi, F., 2016. Genome-wide association between single nucleotide polymorphisms with beef fatty acid profile in Nellore cattle using the single step procedure. BMC Genom. 17, 1-16.

Liu, G.E., Hou, Y., Zhu, B., Cardone, M.F., Jiang, L., Cellamare, A., et al., 2010. Analysis of copy number variations among diverse cattle breeds. Genome Res. 20, 693-703. http://dx.doi.org/10.1101/gr.105403.110. (pmid:20212021).

Liu, G.E., Van Tassell, C.P., Sonstegard, T.S., LI, R.W., Alexander, L.J., Keele, J.W., Matukumalli, L.K., Smith, T.P., Gasbarre, L.C., 2008. Detection of germline and somatic copy number variations in cattle. Dev. Biol. 132, 231-237.

Liu, J., Lingyang, Zhang L., Hangxing, X., LU, R., Zhang, X. Zhang, Zhou, S., Wei, X., Zhao, C., F. DU, L., 2013. Analysis of copy number variation in the sheep genome using 50 K SNP BeadChip array. BMC Genom. 14, 229.

MacHugh, D.E., Shriver, M.D., Loftus, R.T., Cunningham, P., Bradley, D.G., 1997. Microsatellite DNA variation and the evolution, domestication and phylogeography of taurine and zebu cattle (Bos taurus and Bos indicus). Genetics 146 (3), 1071-1086.

Machugh, D.E., Shriver, Md, Rt, Loftus, Cunningham, P., Bradley, Dg, 1997b. Microsatellite DNA variation and the evolution, domestication and phylogeography of taurine and zebu cattle (Bos taurus and Bos indicus). Genetics 146 (3), 1071-1086.

Matukumalli, L.K., Lawley, C.T., Schnabel, R.D., Taylor, J.F., Allan, M.F., Heaton, M.P., O'connell, J., Moore, S.S., Smith, T.P.L., Sonstegard, T.S., 2009. Development and characterization of a high density SNP genotyping assay for cattle. PLoS One 4, 5350.

Nguyen, D.Q., Webber, C., Ponting, C.P., 2006. Bias of selection on human copy-number variants. PLoS Genet. 2, e20. http://dx.doi.org/10.1371/journal.pgen.0020020.

O'Kelly, F.C., 1980. Parasitism and blood composition in genetially different types of cattle grazing in a tropical environment. Vet. Parasitol. 6, 381-390.

G.A. Oliveira Júnior J.P. Eler J. Ramírez-Díaz J.B.S. Ferraz M.L. Santana Inclusion of weaning management group as a random effect in the genetic evaluation of postweaning traits in Nellore cattle Trop. Anim. Health Prod. 4620141031 1036.(v).

Paudel, Y., Madsen, O., Megens, H.-J., Frantz, L., Bosse, M., Bastiaansen, J., et al., 2013. Evolutionary dynamics of copy number variation in pig genomes in the context of adaptation and domestication. BMC Genom. 14, 449.

Paudel, Y., Madsen, O., Megens, H.J., Frantz, L.A.F., Bosse, M., RPMA, Crooijmans, MAM, Groenen, 2015. Copy number variation in the speciation of pigs: a possible prominent role for olfactory receptors. BMC Genom. 16, 330.

D. Pinto K. Darvishi X.H. Shi D. Rajan D. Rigler T. Fitzgerald A.C. Lionel B. Thiruvahindrapuram J.R. MacDonald R. Mills et al. 2011.

Redon, R., Ishikawa, S., Fitch, K.R., Feuk, L., Perry, G.H., Andrews, T.D., Fiegler, H., Shapero, M.H., Carson, A.R., Chen, W., Cho, E.K., Dallaire, S., Freeman, J.L., Gonzalez, J.R., Gratacos, M., Huang, J., Kalaitzopoulos, D., Komura, D., Macdonald, J.R., Marshall, C.R., Mei, R., Montgomery, L., Nishimura, K., Okamura, K., Shen, F., Somerville, M.J., Tchinda, J., Valsesia, A., Woodwark, C., Yang, F., Zhang, J., Zerjal, T., Zhang, J., Armengol, L., Conrad, D.F., Estivill, X., Tyler-Smith, C., Carter, N.P., Aburatani, H., Lee, C., Jones, K.W., Scherer, S.W., Hurles, M.E., 2006. Global variation in copy number in the human genome. Nature 444, 444-454.

Rohrer, G.A., Baker, J.F., Long, C.R., Cartwright, T.C., 1988a. Productive longevity of first-cross cows produced in a fivebreed diallel: I. Reasons for removal. J. Anim. Sci. 66, 2826.

Rohrer, G.A., Baker, J.F., Long, C.R., CartWright, T.C., 1988b. Productive longevity of first-cross cows produced in a five-breed diallel: II. Heterosis and general combining ability. J. Anim. Sci. 662836.

Rosengren-Pielberg, G., Golovko, A., Sundstro“m, E., 2008. A cis-acting regulatory mutation causes premature hair graying and susceptibility to melanoma in the horse. Nat. Genet. 40, 1004-1009.

Salmon Hillbertz, N.H., Isaksson, M., Karlsson, E.K., 2007. Duplication of FGF3, FGF4, FGF19 and ORAOV1 causes hair ridge and predisposition to dermoid sinus in Ridgeback dogs. Nat. Genet. 39, 1318-1320.

Salomón-Torres, R., González-Vizcarra, V.M., Medina-Basulto, G.E., Montaño-Gómez, M.F., Mahadevan, P., Yaurima-Basaldúa, V.H., Villa-Angulo, C., Villa-Angulo, R., 2015. Genome-wide identi cation of copy number variations in Holstein cattle from Baja California, Mexico, using high-density SNP genotyping arrays. Genet. Mol. Res. 14 (4), 11848-11859.

Salomon-Torres, R., Villa-Angulo, R., Villa-Anguloc, C., 2016. Analysis of copy number variations in Mexican Holstein cattle using axiom genome-wide Bos 1 array. Genom. Data $7,97-100$.

Samuelsson, B., 1991. Arachidonic acid metabolism: role in inflammation. Z. Rheumatol. 50 (Suppl 1), 3-6.

Scherer, S.W., Lee, C., Birney, E., Altshuler, D.M., Eichler, E.E., Carter, N.P., Hurles, M.E., Feuk, L., 2007. Challenges and standards in integrating surveys of structural variation. Nat. Genet. 39, S7-S15.

Seroussi, E., Glick, G., Shirak, A., Yakobson, E., Weller, J.I., Ezra, E., et al., 2010. Analysis of copy loss and gain variations in Holstein cattle autosomes using BeadChip SNPs. BMC Genom. 11, 673. http://dx.doi.org/10.1186/1471-2164-11-673. 
(pmid:21114805).

Stafuzza, N.B., Zerlotini, A., Lobo, F.P., Yamagishi, M.E., Chud, T.C., Caetano, A.R., Munari, D.P., Garrick, D.J., Machado, M.A., Martins, M.F., Carvalho, M.R., Cole, J.B., Barbosa da Silva, M.V., 2017. Single nucleotide variants and InDels identified from whole-genome re-sequencing of Guzerat, Gyr, Girolando and Holstein cattle breeds. PLoS One 21 (3), 12.

Stothard, P., Choi, J.-L., Basu, U., Sumner-Thomson, J.M., Meng, Y., Liao, X., Moore, S.S., 2011. Whole genome resequencing of black Angus and Holstein cattle for SNP and CNV discovery. BMC Genom. 12, 559.

Tattini, L., D'aurizio, R., Magi, A., 2015. Detection of genomic structural variants from next-generation sequencing data. Front. Bioeng. Biotechnol. 3.

Tsuang, D.W., Millard, S.P., Ely, B., Chi, P., Wang, K., 2010. The effect of algorithms on copy number variant detection. PLoS One 5, e14456.

Wang, K., Li, M., Hadley, D., Liu, R., Glessner, J., Grant, S.F., Hakonarson, H., Bucan, M., 2007. PennCNV: an integrated hidden Markov model designed for high-resolution copy number variation detection in whole-genome SNP genotyping data. Genome Res. 1665-1674.
Wang, K., Li, M., Hadley, D., Liu, R., Glessner, J., Grant, S.F.A., Hakonarson, H., Bucan, M., 2007. PennCNV: an integrated hidden Markov model designed for highresolution copy number variation detection in whole-genome SNP genotyping data. Genome Res. 17, 1665-1674.

Wang, X., Nahashon, S., Feaster, T.K., Bohannon-Stewart, A., Adefope, N., 2010. An initial map of chromosomal segmental copy number variations in the chicken. BMC Genom. $11,351$.

Winchester, L., Ragoussis, Yau C., 2009. Comparing CNV detection methods for SNP arrays. Brief. Funct. Genom. Prote. 8, 353-366.

Zhang, L., Jia, S., Yang, M., Xu, Y., Li, C., Sun, J., Huang, Y., Lan, X., Lei, C., Zhou, Y., et al., 2014. Detection of copy number variations and their effects in Chinese bulls. BMC Genom. 15, 480.

Zhou, Y., Utsunomiya, Y.T., Xu, Y., Hay, E.H.A., Bickhart, D., Alexandre, P.A., Rosen, B.D., Schroeder, S.G., Carvalheiro, R., Neves, H.H.R., Sonstegard, T.S., Tassell, C.P.V., Ferraz, J.B.S., Fukumasu, Heidge, Garcia, F.J., Liu, G.E., 2016. Genome-wide CNV analysis reveals variants associated with growth traits in Bos indicus. BMC Genom. 17, 419. 